Article

\title{
Crystal Structure of a Variant PAM2 Motif of LARP4B Bound to the MLLE Domain of PABPC1
}

\author{
Clemens Grimm*, Jann-Patrick Pelz, Cornelius Schneider, Katrin Schäffler and Utz Fischer
}

Department of Biochemistry, Theodor Boveri Institute, Biocenter of the University of Würzburg, 97070 Würzburg, Germany; jann-patrick.pelz@msd.de (J.-P.P.); cornelius.schneider@uni-wuerzburg.de (C.S.); katrin.schaeffler@uni-wuerzburg.de (K.S.); utz.fischer@biozentrum.uni-wuerzburg.de (U.F.)

* Correspondence: clemens.grimm@biozentrum.uni-wuerzburg.de

Received: 3 April 2020; Accepted: 4 June 2020; Published: 6 June 2020

\begin{abstract}
Eukaryotic cells determine the protein output of their genetic program by regulating mRNA transcription, localization, translation and turnover rates. This regulation is accomplished by an ensemble of RNA-binding proteins (RBPs) that bind to any given mRNA, thus forming mRNPs. Poly(A) binding proteins (PABPs) are prominent members of virtually all mRNPs that possess poly(A) tails. They serve as multifunctional scaffolds, allowing the recruitment of diverse factors containing a poly(A)-interacting motif (PAM) into mRNPs. We present the crystal structure of the variant PAM motif (termed PAM2w) in the N-terminal part of the positive translation factor LARP4B, which binds to the MLLE domain of the poly(A) binding protein $\mathrm{C} 1$ cytoplasmic 1 (PABPC1). The structural analysis, along with mutational studies in vitro and in vivo, uncovered a new mode of interaction between PAM2 motifs and MLLE domains.
\end{abstract}

Keywords: PAM2w; PAM2; PABC1; MLLE domain; PABP; Poly(A) binding protein

\section{Introduction}

In higher eukaryotes, posttranscriptional mechanisms contribute significantly to gene expression. The mRNA, which is translated into proteins by the ribosomes, has first to undergo several maturation steps, from the primary pre-mRNA transcript to the mature mRNA. These include pre mRNA splicing, capping and polyadenylation, which are a prerequisite for the recruitment of additional factors, which contribute to the formation of the functional messenger ribonucleoprotein particle (mRNP). The vast majority of cellular mRNA is polyadenylated on its $3^{\prime}$-end, allowing binding of poly(A) binding proteins (PABPs). These proteins in turn serve as multifunctional scaffolds, enabling the recruitment of diverse factors to mRNPs [1,2]. PABPs typically contain four consecutive RNA recognition motifs (RRMs 1-4), the two N-terminal RNA recognition motif (RRMs) being necessary and sufficient for binding to the poly(A) tails of mRNAs [3-6]. The C-terminal part, in contrast, contains an unstructured, proline-rich region of low conservation [7-11] and a highly conserved domain termed MLLE (Mademoiselle), characterized by its invariant KITG (Lysine, Isoleucine, Threonine, Glycine) MLLE signature motif [12]. A large number of proteins interacting with PABP-MLLE have been identified, including several translation factors, such as eIF4G [13], Paip1 [14], Paip2 [15] and eRF3 [15]. These proteins contact PABP via a common motif of 12-15 amino acid residues, known as PABP interacting motif 2 (PAM2) [16,17].

Several structures of PAM2 motifs bound to MLLE domains have recently been determined [15,18-22]; see [17] for a review. Within those structures, the respective MLLE domain appears as a bundle of four or five $\alpha$-helices. Two pockets formed in between helix 2 and 3, as well as helix 3 and 5, respectively, are major determinants for the PAM2 interaction. 
The La-related protein (LARP) family comprises five major groups [23-25], whose common structural hallmark is the La module, a combination of the La motif and an adjacent RRM. Structural [26-28] and biochemical studies on the genuine La protein revealed the specific binding of the La module to $3^{\prime}$ oligo-uridylic acid stretches [29,30]. Accordingly, La associates predominantly with polymerase III transcripts in vivo, and influences their stability and/or maturation pathways [31]. LARP7 family members likewise bind the $3^{\prime}$ end of Pol III transcripts. However, they have apparently developed specificity for a subset of Pol III RNA targets [32-34], and facilitate specific functions, such as the stabilization of 7SKRNA or the 2'O methylation of the spliceosomal U6 small nuclear ribonucleic acid (snRNA) [35-39]

In contrast, other LARP members, including 1, 4 and 6, interact with mRNA rather than with Pol III transcripts [40-43]. Moreover, with the exception of LARP6, these cytoplasmic LARP proteins all harbour PAM2 motifs, and likely bind PABPC1 [44-47]. Within this group, LARP4 and LARP4B stand apart, as they harbor a variant PAM2 motif close to their N-termini (termed PAM2w), in which a conserved Tyrosine residue is replaced by Tryptophan [48]. The crystal structure of the LARP4 PAM2w motif, bound to the PABC MLLE domain [49], shows that the PAM2w Tryptophan residue binds in the same pocket on the MLLE surface that harbors the paralogous Tyrosine residue, as seen in structures of MLLE with the classical PAM2 motif.

Here we describe the structural analysis of the interaction of LARP4B with the MLLE domain of PABC. We have shown previously that LARP4B is associated with the ribosome-associated receptor for activated C kinase 1 (RACK1), as well as PABPC1 [47], and that it might act as a stimulatory factor in translation [43]. More recent evidence suggests that the observed increase in translational output upon overexpression of LARP4B can be attributed, at least in part, to an increase in Poly(A) tail length and a stabilization of the transcripts (Mattijssen et al. 2017).

We show that the LARP4B PAM2w motif binds MLLE in the canonical binding cleft, albeit with a divergent binding mode, with respect to the conformation of the PAM2w Tryptophane residue.

\section{Materials and Methods}

\subsection{MLLE Protein Expression, Purification and Complex Crystallization}

The MLLE domain (amino acids 543-621) of human PABPC1 was cloned into vector pETM-30, and expressed in Escherichia coli strain BL21 (DE3) pLysS Rosetta2. The LARP4B-PAM2w peptide was custom-synthesized and purchased from PSL GmbH (Heidelberg, Germany). Crystals were grown with the hanging drop vapor diffusion method by mixing $3 \mu \mathrm{L}$ of MLLE/peptide solution (100 mM sodium chloride, $2 \mathrm{mM}$ s-mercaptoethanol, $10 \mathrm{mM}$ HEPES, $\mathrm{pH} 7.5$ at a concentration of $43 \mathrm{~g} / \mathrm{L}$ supplemented with LARP4B-PAM2w peptide in a 1:1.5 molar ratio) with $3 \mu \mathrm{L}$ of reservoir solution (1.5 M magnesium sulfate, $0.1 \mathrm{M}$ Bis-Tris, $\mathrm{pH}$ 6.5).

\subsection{Crystallographic Methods}

A diffraction dataset for a single crystal was collected at beamline ID14-4 of the European Synchrotron Radiation Facility (ESRF). Data processing and scaling were carried out using the program XDS [50]. The structure was solved by molecular replacement with the program PHASER [51], using the coordinates of unliganded MLLE [15] (PDB entry 3KUR) as a search model. After manual building of the peptide, the model was refined with REFMAC5 [52]. Model coordinates and the diffraction dataset were deposited within the Protein Data Bank (PDB) as entry 3PTH. Data collection and refinement statistics are given in Table 1. 
Table 1. Crystallographic data collection and refinement statistics.

\begin{tabular}{|c|c|}
\hline \multicolumn{2}{|c|}{ Data Collection } \\
\hline Space group & $\mathrm{P} 22_{1} 2_{1}$ \\
\hline Cell dimensions $\mathrm{a}, \mathrm{b}, \mathrm{c}[\AA]$ & $29.15,57.19,59.51$ \\
\hline Resolution $[\AA]$ (outer shell) & $41.2-1.7(1.8-1.7)$ \\
\hline $\mathrm{I} /$ sigma(I) (outer shell) & $14.4(2.4)$ \\
\hline Completeness [\%] (outer shell) & $96.9(86.8)$ \\
\hline Redundancy (outer shell) & $3.2(2.4)$ \\
\hline Wilson B-factor $\left[\AA^{2}\right]$ & 26.1 \\
\hline \multicolumn{2}{|c|}{ Refinement } \\
\hline Resolution [A]] & $41.2-1.7$ \\
\hline No. of reflections & 10805 \\
\hline $\mathrm{R}_{\text {work }} / \mathrm{R}_{\text {free }}$ & $17.2 / 21.8$ \\
\hline No. atoms & 769 \\
\hline Water molecules & 82 \\
\hline \multicolumn{2}{|c|}{$B$-factors $\left[\AA^{2}\right]$} \\
\hline overall & 22.2 \\
\hline MLLE (Chain A) & 19.2 \\
\hline Peptide (chain B) & 24.0 \\
\hline Solvent (chain Z) & 40.3 \\
\hline \multicolumn{2}{|c|}{ RMS (root-mean-square deviation) deviations from ideal } \\
\hline Bond lengths $[\AA ̊]$ & 0.017 \\
\hline Bond angles $\left({ }^{\circ}\right)$ & 1.6 \\
\hline \multicolumn{2}{|c|}{ Ramachandran statistics, residues in [\%] } \\
\hline Most favoured regions & 96.9 \\
\hline Additionally allowed regions & 3.1 \\
\hline Generously allowed regions & 0 \\
\hline
\end{tabular}

\subsection{Cloning, Mutagenesis and Antibodies}

For in vitro translation of $\left[{ }^{35} \mathrm{~S}\right]$-labelled proteins, the respective cDNAs were cloned into expression vector pHA (Invitrogen, Darmstadt, Germany). The luciferase expression vectors pGL3 and pRL-TK were purchased from Promega (Madison, USA) [53]. In vitro translated proteins were produced using the TnT-T7 quick coupled transcription/translation system (Promega, Madison, WI, USA). Site-directed mutagenesis was performed with the Quickchange Site-Directed Mutagenesis Kit (Stratagene, Kirkland, WA, USA) according to the manufacturer's instructions. Antibodies used were obtained as described previously [47].

\subsection{Cell Culture and Luciferase Assay}

HEK293 as well as HeLa cells were grown in DMEM containing 10\% (v/v) FCS and penicillin/streptomycin. To analyze translation in vivo, LARP4B or mutant LARP4B, each cloned into vector $\mathrm{pHA}$, were co-transfected with luciferase expression vectors (pGL3 and pRL-TK) into HEK293 cells using Nanofectin (PAA, Ontario, CA, USA). $48 \mathrm{~h}$ post transfection, cell extracts were prepared [47] and luciferase activity was determined using a standard chemoluminescence detection procedure together with a dual luciferase reporter assay system according to the manufacturer's instructions (Promega, Madison, USA).

\subsection{Immunological Procedures and In Vitro Binding Studies}

Immunoprecipitations and the in vitro binding assay were performed as described previously [37,47]. Proteins were separated by sodium dodecyl sulfate polyacrylamide gel electrophoresis (SDS-PAGE) 
and visualized by Western blotting [54] or analysed by autoradiography [55], respectively. Immunocytochemistry and stress treatment were carried out as described [56].

\section{Results}

\subsection{LARP4B Contains a Variant PAM2 Motif Necessary for Efficient PABC1 Binding}

Our previous in vitro binding experiments, with recombinant LARP4B, demonstrated a direct interaction of PABPC1 with LARP4B [47]. On the LARP4B side, two modules in its N-terminus (amino acids 1-153) and C-terminus (amino acids 308-738) were defined as independent binding modules (Figure 1A). This prompted us to examine the corresponding sequences for possible interaction motifs. Via multiple sequence alignment and a motif search, we discovered a sequence stretch near the N-terminus of LARP4B (residues 56 to 63) that was closely related to the PAM2 motif (Figure 1B). However, the motif found in LARP4B differs from the canonical PAM2 consensus sequence LNxxAxEFxP [17], in that it contains a tryptophan, instead of an otherwise highly conserved phenylalanine residue, at position 10 of the consensus sequence. The phenylalanine residue in this position is a main determinant of the PAM2/MLLE interaction, as can be predicted from all complex structures known so far. The sequence in LARP4B represents a variant PAM2 motif, termed PAM2w $[48,49]$, that can interact with the MLLE domain of PABC1.

To test this for LARP4B, we co-translated and $\left[{ }^{35} \mathrm{~S}\right]$-methionine-labeled PABPC1, along with either a wild-type LARP4B protein or a mutant thereof, in which the variant tryptophan (position 63 in the human protein) had been replaced by lysine. We predicted that this substitution would interfere with the binding of LARP4B to MLLE, as a corresponding substitution in canonical PAM2 motifs is expected to be sterically incompatible with this interaction. The co-translated proteins were co-immunoprecipitated with antibodies against LARP4B, and analyzed by autoradiography (Figure 1C). Whereas the wild-type protein formed a stoichiometric complex with PABPC1, binding to the mutant version was markedly reduced (compare lanes 1 and 3). No proteins were immunoprecipitated by a control serum, illustrating the specificity of the antibody used (lanes 2 and 4). Next, we tested whether the same effect was observable in vivo. Plasmids encoding the wild-type and mutant LARP4B protein were co-transfected, with PABPC1-encoding plasmids, into HEK293 cells. After this, lysis immunoprecipitations were carried out as described above, and the proteins were detected by Western blotting. Mutant LARP4B bound slightly more weakly to PABPC1, as compared to the wild type. However, this effect seemed to not be as strong as in vitro. These results gave us a first hint that the PAM2 $w$ domain might contribute to the interaction of LARP4B with PABPC1 in vitro, but that in vivo additional factors or other regions in LARP4B could likely influence binding.

\subsection{Crystal Structure of the LARP4B PAM2w/MLLE Complex}

In order to understand the molecular details of the uncovered interaction, we co-crystallized a peptide representing the LARP4B PAM2w motif (residues 56 to 70), together with the PABC1 MLLE domain (residues 543 to 621), and solved the crystal structure. Figure 2 shows the molecular details of the peptide/protein interaction. 
A

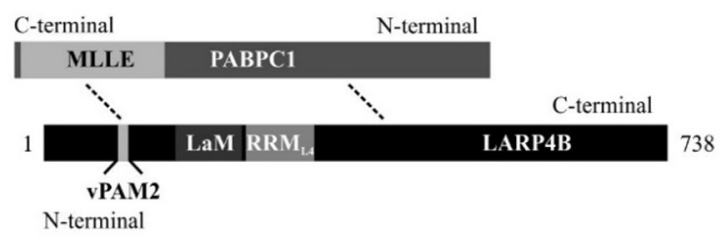

B

\begin{tabular}{|c|c|c|c|}
\hline Hs & eRF3/GSPT1 N-PAM & LNVNAKPF & VPNVHAAEFVPSFLRGPTQP \\
\hline Hs & eRF3/GSPT1 C-PAM & PNVHAAEF & VPSFLRGPTQPPTLPAGSGS \\
\hline $\mathrm{Dm}$ & eEF1-like protein & LNVNAVEF & VPSFSYNSVVNVAEEAAAVV \\
\hline Hs & TTC3 1207 & LNPAAREF & KPDVKSKPVSDSSSAPAFEN \\
\hline At & ERD15 & LNPDAPLF & IPAAVRQVEDFSPEWWQLVT \\
\hline Hs & USP10 & APEF & ILGCTASKITPDGITKEASY \\
\hline At & Paip2 & APVF & DPVEFREVEDFSPKWWDLVT \\
\hline $\mathrm{Dm}$ & Paip2 & $A D E F$ & VPRCHVIDFPAS-------- \\
\hline Hs & Ataxin-2 & AKEF & NPRSESQPKPSTTPTSPRPQ \\
\hline & Paipl & PEF & YPSGYSSSYTESYEDGCEDY \\
\hline & LARP 4 & LNPNAKVW & QEIAPGNTDATPVTHGTESS \\
\hline ( & LARP 4B & LNPNAEVW & GAPVLHLEASSAADGVSAAW \\
\hline & GW182 & WPPEF & HPGVPWKGLQ \\
\hline
\end{tabular}

C

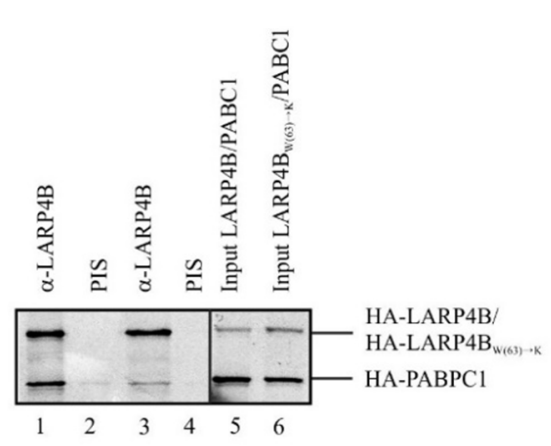

D

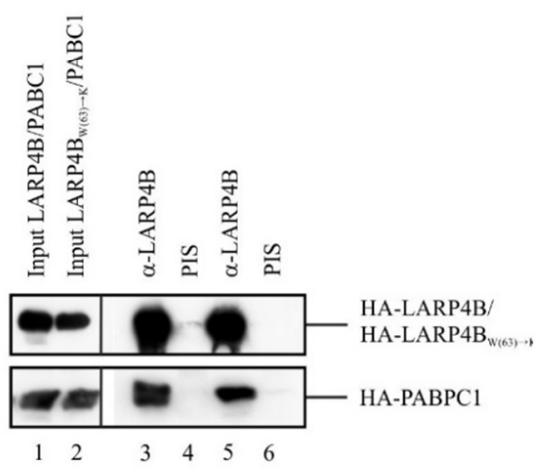

Figure 1. Characterisation of the PAM2 $w$ motif and its binding to poly $(\mathrm{A})$ binding protein $\mathrm{C} 1$ cytoplasmic 1 Mademoiselle domain (PABPC1-MLLE). (A) A schematic model of the LARP4B-PABPC1 interaction. (B) Sequence alignment of PAM2 motifs of several proteins from different organisms (Hs-Homo sapiens; Dm-Drosophila melanogaster; At-Arabidopsis thaliana): Hs eRF3 (elongation release factor 3), Hs TTC3 (E3 ubiquitin-ligase TTC3), Hs Ataxin-2, Paip1 [Poly(A) interacting protein 1], Hs LARP4 and LARP4B (La-related proteins 4 and 4B), Dm eEF1-like protein (eukaryotic elongation factor 1-like protein), Dm Paip2 [Poly(A) interacting protein 2], At. ERD15 (early response to dehydration 15), At. Paip2 [Poly(A) interacting protein 2]. Conserved amino acids are highlighted by bold characters, the variant tryptophan residues of LARP4 and LARP4B are underlined. (C) In vitro binding experiment using protein-G-Sepharose coupled antibody against LARP4B (lanes 1 and 3) or a pre-immune serum (lanes 2 and 4). HA-PABPC1, as well as HA-LARP4B or HA-LARP4B $(\mathrm{W} 63) \rightarrow \mathrm{K}$, respectively, were co-translated in vitro, $\left[{ }^{35} \mathrm{~S}\right]$-labeled and immunoprecipitated with the indicated antibodies. Bound proteins were detected by autoradiography (HA-LARP4B lanes 1-2, HA-LARP4B $\mathrm{B}_{(\mathrm{W} 63) \rightarrow \mathrm{K}}$ lanes 3-4). Input, $5 \%$ of $\left[{ }^{35} \mathrm{~S}\right]$-labeled HA-PABPC1, HA-LARP4B (lane 5) or HA-LARP4B $\mathrm{B}_{(\mathrm{W} 63) \rightarrow \mathrm{K}}$ (lane 6). (D) Immunoprecipitation of HEK293 cell extracts overexpressing either HA-LARP4B or $\mathrm{HA}-\mathrm{LARP} 4 \mathrm{~B}_{\mathrm{W}(63) \rightarrow \mathrm{K}}$ using an antibody against LARP4B (lanes 3 and 5) or a pre-immune serum (lanes 2 and 4). Lanes 1 and 2 show the transfection controls. The gel was analyzed by Western blotting. 


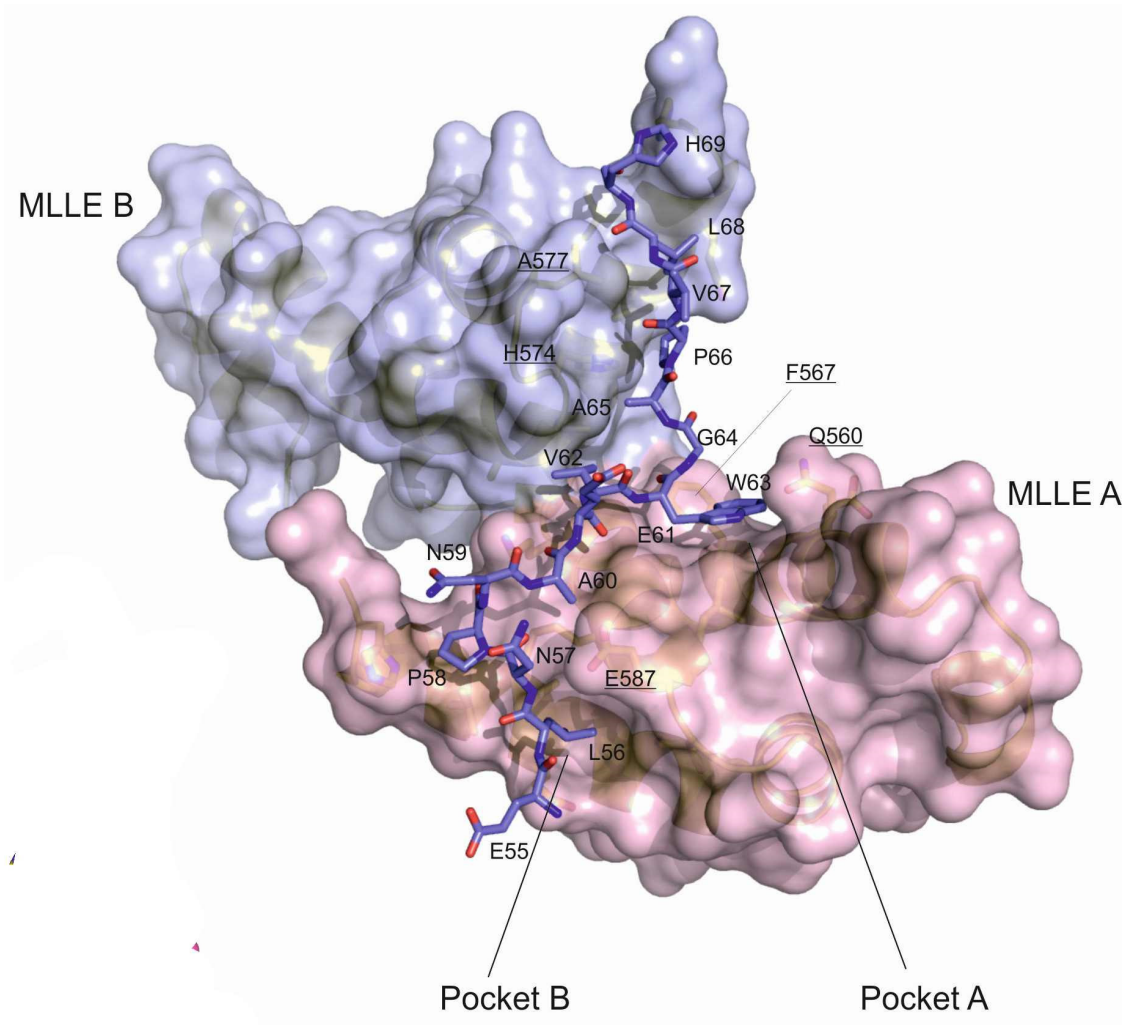

Figure 2. Surface representation of MLLE molecule A (pink) and B (blue) with the bound LARP4B-PAM2w peptide, shown as a stick model. MLLE residue names are underlined. (blue) and $C$ (green).

Within the crystal structure, a single LARP4B-PAM2w complex contacts a neighbouring, symmetry-related MLLE entity, providing an extended interface that creates a deep cleft in the interface region into which the peptide binds, running over or in between the surfaces of both symmetry-related MLLE molecules (Figure 2). Binding of the N-terminal, the canonical part of the LARP4B-PAM2w peptide, is concordant with the structures reported for other PAM2 motifs bound to MLLE (see text below and also Figure 3). A hydrophobic binding pocket (A) in between helix $\alpha 2$ and $\alpha 3$, lined by residues Q560, E564, L566, F567, K580, T582 and L586, harbours W63 of the LARP4B-PAM2w motif (Figure 2). This demonstrates the ability of pocket A to either bind a tryptophan, or alternatively, a less bulky phenylalanine residue. This behavior conforms to the MLLE conserved binding sequence pattern reported previously [17]. A second hydrophobic pocket (B) lies between helix $\alpha 3$ and $\alpha 5$, outlined by residues L569, T576, L597, P600 and L603, harbouring L56 of the PAM2w motif. In between peptide residues L56 and W63, a $\beta$-turn is stabilized by P58. While A60 is accommodated by a shallow pocket on the surface of MLLE molecule A [crystallographic symmetry operation $(x, y, z)$ ], E55, N57, N59 and E61, the remaining hydrophilic residues of the canonical PAM2w motif, point away from the MLLE A surface into the solvent. The C-terminal, a variable part of the LARP4B-PAM2w peptide, is located within the gap between the contacting symmetry-related MLLE molecules B [symmetry operation $(x-1, y, z)$ ] and $C$ [symmetry operation $(x-0.5,-y+0.5,-z)$ ]. To enter this gap, the peptide performs an almost $90^{\circ}$ turn around G64. Inside, V67 binds into a hydrophobic pocket located on the MLLE B surface, interacting mainly with L569 and P600. The neighbouring residue, Leu68, binds into a hydrophobic pocket on MLLE C, outlined by L577, I581, V611 and L614. Well-defined electron density was observed for the whole peptide, and only the C-terminal residue, L70, was disordered and therefore omitted from the model.

Altogether, a total continuous surface of $1621 \AA^{2}$, or $33.3 \%$ of a single MLLE molecule's accessible surface area, is buried within the MLLE-MLLE and MLLE-peptide contacts. The result of these 
interactions is an extended and rugged interface, centred about the LARP4B-PAM2w peptide. This is reflected in a zone of comparably low B-factors within this region.

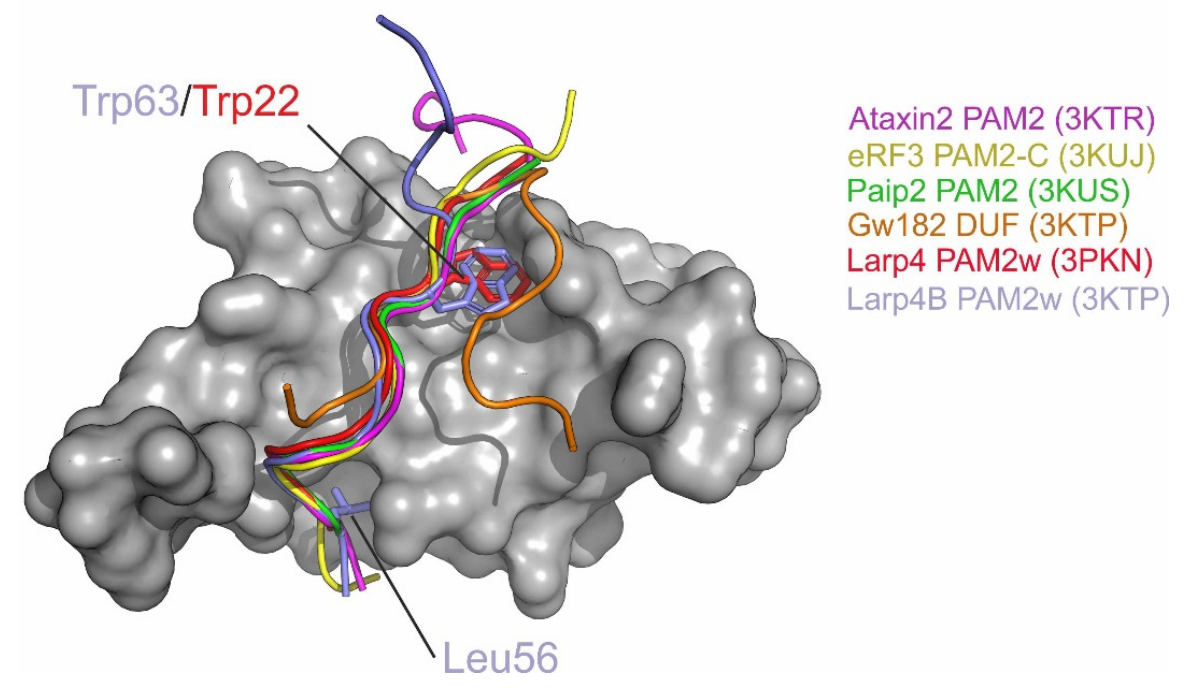

Figure 3. Superposition of the C $\alpha$-trace of LARP4B-PAM2w (blue, sidechains L56 and W63 shown) with $C \alpha$-traces of the LARP4-PAM2w (PDB ID 3PKN, red, sidechain W22 shown) and PAM2 peptides from PABP interacting protein (Paip2, PDB ID 3KUS, green), release factor 3 (eRF3 C-terminal motif, PDB ID 3KUJ, yellow) and Ataxin2 (PDB ID 3KTR, pink). The GW182 DUF peptide (3KTP) is depicted in orange.

\subsection{PAM2w/PAM2 Sequences Can Be Divided into a Canonical and a Variable Part}

A superposition of the MLLE co-structures, with PAM2 or PAM2w motifs from LARP4B, LARP4, Ataxin2, eRF3 (N-terminal as well as C-terminal PAM2 motif) and Paip2, clearly illustrates the bipartite character of the PAM2-MLLE interactions (Figure 3). The C-terminal, the canonical parts of the bound PAM2 peptides, overlap closely, exhibiting the same mode of binding to the MLLE surface within all structures. The main anchoring points for the binding of the canonical motif parts are, in all cases, the two hydrophobic pockets A and B. In between those, all peptides display a $\beta$-turn, which is stabilized by a hydrogen bond between the conserved PAM2-asparagine LNxxAxx[F/W] (position 4 of the consensus sequence, corresponding to Asn57 of LARP4B) preceding the turn, and the backboncensus sequence (LNxxAxx[F/W]). The conserved alanine residue LNxxAxx[F/W] following the turn points towards the MLLE surface, and therefore a bulkier side chain at this position would sterically be highly unfavourable. In the absence of a significant induced fit, the relatively wide and shallow hydrophobic pockets A and B are able to harbor different hydrophobic side chains. Judging from the frequency of occurrence within so-far identified PAM2 motifs, pocket B has a preference for Phe, but is also able to harbor a Trp residue, as shown previously for LARP4 by [49], as well as in this study. Of note, the versatility of this pocket is also reflected by its ability to harbor the bound tryptophane in different sidechain conformations [compare LARP4(Trp22) and LARP4B(Trp63) in Figure 3]. Correspondingly, pocket A seems to prefer Leu, which binds in a radial mode with respect to the protein surface. However, the C-terminal of one of the two overlapping eRF3 PAM2 motifs shows an $\mathrm{F}$ residue binding with a tangential mode into pocket $\mathrm{A}$. While the variable sequence downstream of the canonical motif contributes significantly to the PAM2/MLLE interaction, this region of the corresponding bound peptides runs over completely different parts of the MLLE surface within the different co-structures. Likewise, the GW182 DUF peptide is anchored by its F1389 residue within pocket $\mathrm{B}$, and locally shares a few more interactions in the adjacent regions with PAM2 peptides. However, the rest of the contacts differ largely from those seen for canonical PAM2 sites. 


\subsection{Disruption of the PAM2w/MLLE Interaction Does Not Affect Recruitment of LARP4B to Stress Granules} upon Arsenite Stress

We tested whether the integrity of the PAM2w motif is critical for the function of LARP4B. We showed previously that LARP4B translocates to subcellular domains, termed stress granules (SGs), under various stress stimuli [47]; [57]. It is believed that SGs under these conditions serve as storage pools for stalled, mRNA-bound translation initiation complexes formed upon polyribosome disassembly $[57,58]$. As the PAM2w-MLLE interaction of LARP4B with PABPC1 appeared to have only minor effects on LARB4B binding to PABPC1 in vivo, we set out to test whether the LARP4B would be recruited to the same mRNPs in vivo, upon disruption of the PAM2w-PABPC1 interaction. To this end we investigated whether the $L A R P 4 B_{W(63) \rightarrow K}$ mutant recapitulates our previously observed recruitment of wild type (wt) LARP4B to stress granules upon arsenite treatment [47].

We therefore analyzed the intracellular localization of a HA-tagged LARP4B truncation (the N-terminal part of the protein, including the PAM2w motif), as well as the $\operatorname{LARP} 4 \mathrm{~B}_{\mathrm{W}(63) \rightarrow \mathrm{K}}$ mutant under normal growth and under stress conditions (Figure 4 shows a schematic model of the LARP4B constructs used). Under normal conditions, the LARP4B variants were homogenously distributed in the cytoplasm (Figure 4, panels A-E, K-O and U-Y). As shown in Figure 4, all tested proteins translocated upon stress induction with arsenite to SGs (compare panels F-J, P-T and Z-D'). The Fragile X Mental Retardation Protein (FMRP), a well-established SG marker protein, served as a control in these experiments (see Figure 4, panels B, G, L, Q, V and $A^{\prime}$ ). In sum, these experiments indicate that uncovered interaction, while strongly affecting LARP4B's interaction with PABPC1 in vitro, seems to not be sufficient in vivo to disrupt the recruitment of LARP4B to stress granules. These results suggest that additional factors, until now unknown, contribute to the recruitment of LARP4B to its native mRNPs.
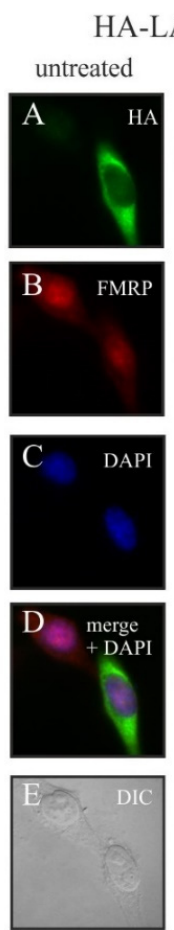

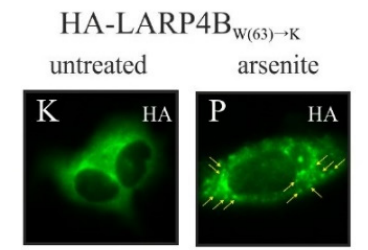

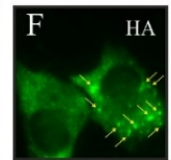
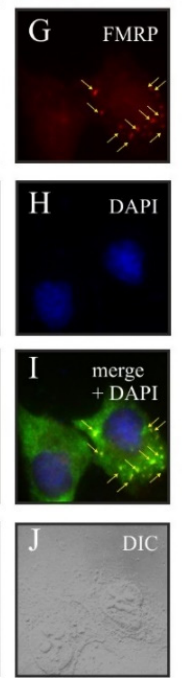
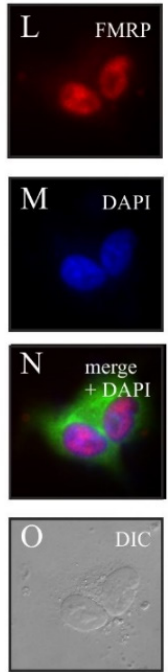
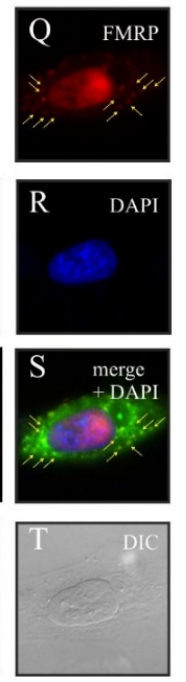

HA-LARP4B-RRM-Ct

untreated
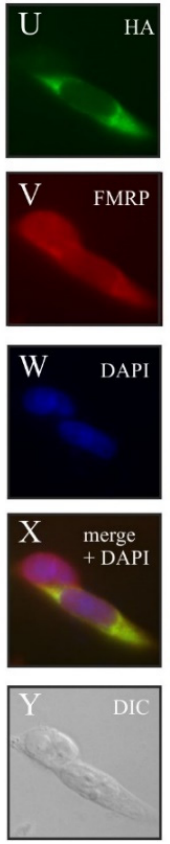

arsenite
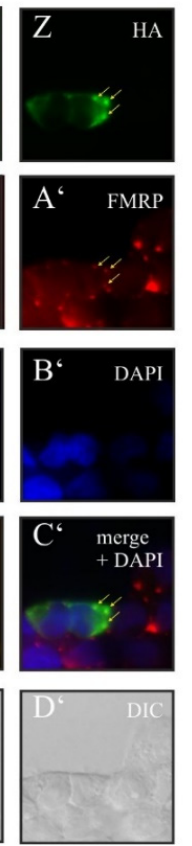

Figure 4. The C-terminus of LARP4B is sufficient to accumulate in stress granules. Upper part, depiction of the LARP4B constructs used for immunofluorescence. Lower part, immunofluorescence studies in HeLa cells transfected with the introduced constructs, using antibodies against Human influenza hemagglutinin (HA) and fragile $X$ mental retardation protein (FMRP) as a stress granules marker protein. Cells were either mock-treated (panels A-E, K-O, U-Y) or treated with arsenite (panels F-J, P-T and Z-D'). SG are marked by arrows. 


\section{Discussion}

A large variety of different proteins are recruited to mRNPs by virtue of their PAM motifs. Accordingly, this interaction has been studied intensely, both at the biochemical and structural level. Here, we have uncovered the atomic details of the LARP4B-PAM2w interaction with the MLLE domain. A comparison of all relevant atomic structures revealed that the major determinants for the binding of PAM2w and PAM2 motifs reside within their canonical part, i.e., in the parts that are shared between both motifs. Therefore, binding of different proteins containing PAM2 or PAM2w motifs is likely to occur mutually exclusively. Given the large number of protein factors containing a PAM2/PAM2w or related motif, they are expected to compete for binding to their respective binding sites located on the PABP-MLLE domain. In this regard, the variable part of PAM2/PAM2w sites, and/or other binding surfaces located in other parts of the protein, might help to fine tune and diversify these interactions, while the canonical part might represent a common 'interaction module', providing a basal affinity. At the border of both motif parts, the $\mathrm{LNxxAxx}[\mathrm{F} / \mathrm{W}] \mathrm{F} / \mathrm{W}$ residue could provide a switch point, sending the variable part of the bound peptide chain to different MLLE surface areas, depending on the different stereo-chemistries imposed by either a $\mathrm{F}$ or a $\mathrm{W}$ residue.

In this study, we observe the binding of the variable part of the PAM2w motif, extruding from the canonical MLLE complex, into a cleft on the surface of a MLLE unit neighbouring in the crystal packing. In this regard it is worth mentioning that the MLLE domain used here failed to form crystals on its own, but rapidly crystallized upon addition of the PAM2 $\mathrm{w}$ peptide. This can be explained by the stable and extended contacts that emerge from the peptide contacting three distinct MLLE molecules. There is currently no data that supports this behavior in vitro, in solution. Nevertheless, one could hypothesize that, in vivo, the PAM2w site, located within the largely unstructured and flexible N-terminus of LARP4B, could simultaneously contact two or more distinct MLLE units of neighbouring, poly(A)-bound PABC molecules. However, it should not go unnoticed that the cross-species sequence conservation is only high in the canonical part of the LARP4B-PAM2w motif, and comparably low in its variable part [59].

Biochemical studies have suggested that LARP4B is recruited by, and stably bound on, mRNPs by virtue of an elaborate protein and mRNA network [47]. It was hence not entirely unexpected that a missense mutation in PAM2w, or even the entire deletion of the motif, failed to interfere with the recruitment of LARP4B to this mRNP. We speculate that the interface between PAM2 $w$ and MLLE contributes only partially to the stable recruitment of LARP4B to mRNPs. Most likely, recruitment of LARP4B to its mRNPs is aided by the C-terminal region, which was shown to co-immunoprecipitate with PABPC1 independently of PAM2w [47]. Alternatively, and not mutually exclusively, our studies may indicate an additional function of LARP4B that critically depends on the intact PAM2w motif. Recently, LARP4B overexpression was shown to increase Poly(A) tail length and increase mRNA stability (Mattijssen et al. 2017). It is tempting to speculate that the disruption of the LARP4B-PAM2w and -MLLE interaction has an influence on this important role of LARP4B, similar to what was shown for the related, but functionally different, LARP4 (Mattijssen et al. 2017).

In addition, it was reported that LARP4 possesses a PAM2 variant similar to that of LARP4B, and the authors of this study presented a crystal structure of the corresponding PAM2w-MLLE complex [49]. This structure features a similar binding mode for the canonical motif part. However, this structure differed from the one we have reported in this manuscript, in that the LARP4-PAM2w peptide bound to one MLLE unit only. This raises the interesting possibility that different PAM2 variants might use alternative modes of interaction.

Author Contributions: Conceptualization, C.G. and U.F.; Data curation, C.G. and J.-P.P.; Formal analysis, C.G., J.-P.P. and K.S.; Funding acquisition, U.F.; Investigation, C.G., J.-P.P., K.S. and U.F.; Project administration, U.F.; Supervision, C.G. and U.F.; Writing-original draft, C.G., J.-P.P., K.S. and U.F.; Writing-review \& editing, C.G., C.S. and U.F. All authors have read and agreed to the published version of the manuscript.

Funding: This study was supported by grant Fi573/15-2 from the Deutsche Forschungsgemeinschaft. 
Acknowledgments: We thank the team at beamline ID14-4 of the European Synchrotron Radiation Facility, Grenoble for their excellent technical support during crystallographic data collection.

Conflicts of Interest: All authors declare that they have no competing interests.

\section{References}

1. Mangus, D.A.; Amrani, N.; Jacobson, A. Pbp1p, a factor interacting with Saccharomyces cerevisiae poly(A)-binding protein, regulates polyadenylation. Mol. Cell. Biol. 1998, 18, 7383-7396. [CrossRef] [PubMed]

2. Mangus, D.A.; Evans, M.C.; Jacobson, A. Poly(A)-binding proteins: Multifunctional scaffolds for the post-transcriptional control of gene expression. Genome Biol. 2003, 4, 223. [CrossRef]

3. Burd, C.G.; Dreyfuss, G. Conserved structures and diversity of functions of RNA-binding proteins. Science 1994, 265, 615-621. [CrossRef] [PubMed]

4. Burd, C.G.; Matunis, E.L.; Dreyfuss, G. The multiple RNA-binding domains of the mRNA poly(A)-binding protein have different RNA-binding activities. Mol. Cell. Biol. 1991, 11, 3419-3424. [CrossRef]

5. Khanam, T.; Muddashetty, R.S.; Kahvejian, A.; Sonenberg, N.; Brosius, J. Poly(A)-binding protein binds to A-rich sequences via RNA-binding domains 1+2 and 3+4. RNA Biol. 2006, 3, 170-177. [CrossRef] [PubMed]

6. Schafer, I.B.; Yamashita, M.; Schuller, J.M.; Schussler, S.; Reichelt, P.; Strauss, M.; Conti, E. Molecular Basis for poly(A) RNP Architecture and Recognition by the Pan2-Pan3 Deadenylase. Cell 2019, 177, 1619-1631.e1621. [CrossRef]

7. Kuhn, U.; Pieler, T. Xenopus poly(A) binding protein: Functional domains in RNA binding and protein-protein interaction. J. Mol. Biol. 1996, 256, 20-30. [CrossRef]

8. Gray, N.K.; Coller, J.M.; Dickson, K.S.; Wickens, M. Multiple portions of poly(A)-binding protein stimulate translation in vivo. EMBO J. 2000, 19, 4723-4733. [CrossRef]

9. Collier, B.; Gorgoni, B.; Loveridge, C.; Cooke, H.J.; Gray, N.K. The DAZL family proteins are PABP-binding proteins that regulate translation in germ cells. EMBO J. 2005, 24, 2656-2666. [CrossRef]

10. Mangus, D.A.; Evans, M.C.; Agrin, N.S.; Smith, M.; Gongidi, P.; Jacobson, A. Positive and negative regulation of poly(A) nuclease. Mol. Cell. Biol. 2004, 24, 5521-5533. [CrossRef]

11. Patel, G.P.; Bag, J. IMP1 interacts with poly(A)-binding protein (PABP) and the autoregulatory translational control element of PABP-mRNA through the KH III-IV domain. FEBS J. 2006, 273, 5678-5690. [CrossRef] [PubMed]

12. Kozlov, G.; Safaee, N.; Rosenauer, A.; Gehring, K. Structural basis of binding of P-body-associated proteins GW182 and ataxin-2 by the Mlle domain of poly(A)-binding protein. J. Biol. Chem. 2010, 285, 13599-13606. [CrossRef] [PubMed]

13. Cheng, S.; Gallie, D.R. eIF4G, eIFiso4G, and eIF4B bind the poly(A)-binding protein through overlapping sites within the RNA recognition motif domains. J. Biol. Chem. 2007, 282, 25247-25258. [CrossRef]

14. Roy, G.; De Crescenzo, G.; Khaleghpour, K.; Kahvejian, A.; O'Connor-McCourt, M.; Sonenberg, N. Paip1 interacts with poly(A) binding protein through two independent binding motifs. Mol. Cell. Biol. 2002, 22, 3769-3782. [CrossRef] [PubMed]

15. Kozlov, G.; Menade, M.; Rosenauer, A.; Nguyen, L.; Gehring, K. Molecular determinants of PAM2 recognition by the MLLE domain of poly(A)-binding protein. J. Mol. Biol. 2010, 397, 397-407. [CrossRef] [PubMed]

16. Lim, N.S.; Kozlov, G.; Chang, T.C.; Groover, O.; Siddiqui, N.; Volpon, L.; De Crescenzo, G.; Shyu, A.B.; Gehring, K. Comparative peptide binding studies of the PABC domains from the ubiquitin-protein isopeptide ligase HYD and poly(A)-binding protein. Implications for HYD function. J. Biol. Chem. 2006, 281, 14376-14382. [CrossRef]

17. Xie, J.; Kozlov, G.; Gehring, K. The "tale" of poly(A) binding protein: The MLLE domain and PAM2-containing proteins. Biochim. Biophys. Acta 2014, 1839, 1062-1068. [CrossRef]

18. Kozlov, G.; Siddiqui, N.; Coillet-Matillon, S.; Trempe, J.F.; Ekiel, I.; Sprules, T.; Gehring, K. Solution structure of the orphan PABC domain from Saccharomyces cerevisiae poly(A)-binding protein. J. Biol. Chem. 2002, 277, 22822-22828. [CrossRef]

19. Deo, R.C.; Sonenberg, N.; Burley, S.K. X-ray structure of the human hyperplastic discs protein: An ortholog of the C-terminal domain of poly(A)-binding protein. Proc. Natl. Acad. Sci. USA 2001, 98, 4414-4419. [CrossRef] 
20. Kozlov, G.; De Crescenzo, G.; Lim, N.S.; Siddiqui, N.; Fantus, D.; Kahvejian, A.; Trempe, J.F.; Elias, D.; Ekiel, I.; Sonenberg, N.; et al. Structural basis of ligand recognition by PABC, a highly specific peptide-binding domain found in poly(A)-binding protein and a HECT ubiquitin ligase. EMBO J. 2004, 23, 272-281. [CrossRef]

21. Tritschler, F.; Huntzinger, E.; Izaurralde, E. Role of GW182 proteins and PABPC1 in the miRNA pathway: A sense of deja vu. Nat. Rev. Mol. Cell. Biol. 2010, 11, 379-384. [CrossRef]

22. Dos Santos Rodrigues, F.H.; Firczuk, H.; Breeze, A.L.; Cameron, A.D.; Walko, M.; Wilson, A.J.; Zanchin, N.I.T.; McCarthy, J.E.G. The Leishmania PABP1-eIF4E4 interface: A novel 5'-3' interaction architecture for trans-spliced mRNAs. Nucleic Acids Res. 2019, 47, 1493-1504. [CrossRef] [PubMed]

23. Bousquet-Antonelli, C.; Deragon, J.M. A comprehensive analysis of the La-motif protein superfamily. RNA 2009, 15, 750-764. [CrossRef]

24. Bayfield, M.A.; Yang, R.; Maraia, R.J. Conserved and divergent features of the structure and function of La and La-related proteins (LARPs). Biochim. Biophys. Acta 2010, 1799, 365-378. [CrossRef] [PubMed]

25. Maraia, R.J.; Mattijssen, S.; Cruz-Gallardo, I.; Conte, M.R. The La and related RNA-binding proteins (LARPs): Structures, functions, and evolving perspectives. Wiley Interdiscip. Rev. RNA 2017, 8. [CrossRef] [PubMed]

26. Alfano, C.; Babon, J.; Kelly, G.; Curry, S.; Conte, M.R. Resonance assignment and secondary structure of an N-terminal fragment of the human La protein. J. Biomol. NMR 2003, 27, 93-94. [CrossRef] [PubMed]

27. Alfano, C.; Sanfelice, D.; Babon, J.; Kelly, G.; Jacks, A.; Curry, S.; Conte, M.R. Structural analysis of cooperative RNA binding by the La motif and central RRM domain of human La protein. Nat. Struct. Mol. Biol. 2004, 11, 323-329. [CrossRef]

28. Dong, G.; Chakshusmathi, G.; Wolin, S.L.; Reinisch, K.M. Structure of the La motif: A winged helix domain mediates RNA binding via a conserved aromatic patch. EMBO J. 2004, 23, 1000-1007. [CrossRef]

29. Stefano, J.E. Purified lupus antigen La recognizes an oligouridylate stretch common to the $3^{\prime}$ termini of RNA polymerase III transcripts. Cell 1984, 36, 145-154. [CrossRef]

30. Wolin, S.L.; Cedervall, T. The La protein. Annu. Rev. Biochem. 2002, 71, 375-403. [CrossRef]

31. Maraia, R.J.; Lamichhane, T.N. 3' processing of eukaryotic precursor tRNAs. Wiley Interdiscip. Rev. RNA 2011, 2, 362-375. [CrossRef] [PubMed]

32. Muniz, L.; Egloff, S.; Kiss, T. RNA elements directing in vivo assembly of the 7SK/MePCE/Larp7 transcriptional regulatory snRNP. Nucleic Acids Res. 2013, 41, 4686-4698. [CrossRef] [PubMed]

33. Eichhorn, C.D.; Yang, Y.; Repeta, L.; Feigon, J. Structural basis for recognition of human 7SK long noncoding RNA by the La-related protein Larp7. Proc. Natl. Acad. Sci. USA 2018, 115, E6457-E6466. [CrossRef]

34. Prathapam, R.; Witkin, K.L.; O'Connor, C.M.; Collins, K. A telomerase holoenzyme protein enhances telomerase RNA assembly with telomerase reverse transcriptase. Nat. Struct. Mol. Biol. 2005, 12, 252-257. [CrossRef] [PubMed]

35. Hasler, D.; Meduri, R.; Bak, M.; Lehmann, G.; Heizinger, L.; Wang, X.; Li, Z.T.; Sement, F.M.; Bruckmann, A.; Dock-Bregeon, A.C.; et al. The Alazami Syndrome-Associated Protein LARP7 Guides U6 Small Nuclear RNA Modification and Contributes to Splicing Robustness. Mol. Cell 2020, 77, 1014-1031 e1016. [CrossRef]

36. Wang, X.; Li, Z.T.; Yan, Y.; Lin, P.; Tang, W.; Hasler, D.; Meduri, R.; Li, Y.; Hua, M.M.; Qi, H.T.; et al. LARP7-Mediated U6 snRNA Modification Ensures Splicing Fidelity and Spermatogenesis in Mice. Mol. Cell 2020, 77, 999-1013 e1016. [CrossRef]

37. Markert, A.; Grimm, M.; Martinez, J.; Wiesner, J.; Meyerhans, A.; Meyuhas, O.; Sickmann, A.; Fischer, U. The La-related protein LARP7 is a component of the 7SK ribonucleoprotein and affects transcription of cellular and viral polymerase II genes. EMBO Rep. 2008, 9, 569-575. [CrossRef]

38. Aigner, S.; Postberg, J.; Lipps, H.J.; Cech, T.R. The Euplotes La motif protein p43 has properties of a telomerase-specific subunit. Biochemistry 2003, 42, 5736-5747. [CrossRef]

39. Stone, M.D.; Mihalusova, M.; O'Connor C, M.; Prathapam, R.; Collins, K.; Zhuang, X. Stepwise protein-mediated RNA folding directs assembly of telomerase ribonucleoprotein. Nature 2007, 446, 458-461. [CrossRef]

40. Mattijssen, S.; Arimbasseri, A.G.; Iben, J.R.; Gaidamakov, S.; Lee, J.; Hafner, M.; Maraia, R.J. LARP4 mRNA codon-tRNA match contributes to LARP4 activity for ribosomal protein mRNA poly(A) tail length protection. Elife 2017, 6. [CrossRef]

41. Fonseca, B.D.; Lahr, R.M.; Damgaard, C.K.; Alain, T.; Berman, A.J. LARP1 on TOP of ribosome production. Wiley Interdiscip. Rev. RNA 2018, e1480. [CrossRef] 
42. Zhang, Y.; Stefanovic, B. LARP6 Meets Collagen mRNA: Specific Regulation of Type I Collagen Expression. Int. J. Mol. Sci. 2016, 17, 419. [CrossRef] [PubMed]

43. Kuspert, M.; Murakawa, Y.; Schaffler, K.; Vanselow, J.T.; Wolf, E.; Juranek, S.; Schlosser, A.; Landthaler, M.; Fischer, U. LARP4B is an AU-rich sequence associated factor that promotes mRNA accumulation and translation. RNA 2015, 21, 1294-1305. [CrossRef] [PubMed]

44. Merret, R.; Martino, L.; Bousquet-Antonelli, C.; Fneich, S.; Descombin, J.; Billey, E.; Conte, M.R.; Deragon, J.M. The association of a La module with the PABP-interacting motif PAM2 is a recurrent evolutionary process that led to the neofunctionalization of La-related proteins. RNA 2013, 19, 36-50. [CrossRef] [PubMed]

45. Fonseca, B.D.; Zakaria, C.; Jia, J.J.; Graber, T.E.; Svitkin, Y.; Tahmasebi, S.; Healy, D.; Hoang, H.D.; Jensen, J.M.; Diao, I.T.; et al. La-related Protein 1 (LARP1) Represses Terminal Oligopyrimidine (TOP) mRNA Translation Downstream of mTOR Complex 1 (mTORC1). J. Biol. Chem. 2015, 290, 15996-16020. [CrossRef] [PubMed]

46. Cruz-Gallardo, I.; Martino, L.; Kelly, G.; Atkinson, R.A.; Trotta, R.; De Tito, S.; Coleman, P.; Ahdash, Z.; $\mathrm{Gu}$, Y.; Bui, T.T.T.; et al. LARP4A recognizes polyA RNA via a novel binding mechanism mediated by disordered regions and involving the PAM2w motif, revealing interplay between PABP, LARP4A and mRNA. Nucleic Acids Res. 2019, 47, 4272-4291. [CrossRef]

47. Schaffler, K.; Schulz, K.; Hirmer, A.; Wiesner, J.; Grimm, M.; Sickmann, A.; Fischer, U. A stimulatory role for the La-related protein 4B in translation. RNA 2010, 16, 1488-1499. [CrossRef]

48. Dock-Bregeon, A.C.; Lewis, K.A.; Conte, M.R. The La-related proteins: Structures and interactions of a versatile superfamily of RNA-binding proteins. RNA Biol. 2019, 1-16. [CrossRef]

49. Yang, R.; Gaidamakov, S.A.; Xie, J.; Lee, J.; Martino, L.; Kozlov, G.; Crawford, A.K.; Russo, A.N.; Conte, M.R.; Gehring, K.; et al. La-related protein 4 binds poly(A), interacts with the poly(A)-binding protein MLLE domain via a variant PAM2w motif, and can promote mRNA stability. Mol. Cell. Biol. 2011, 31, 542-556. [CrossRef]

50. Kabsch, W. Xds. Acta Crystallogr D Biol. Crystallogr 2010, 66, 125-132. [CrossRef]

51. McCoy, A.J.; Grosse-Kunstleve, R.W.; Adams, P.D.; Winn, M.D.; Storoni, L.C.; Read, R.J. Phaser crystallographic software. J. Appl. Crystallogr. 2007, 40, 658-674. [CrossRef] [PubMed]

52. Murshudov, G.N.; Vagin, A.A.; Dodson, E.J. Refinement of macromolecular structures by the maximum-likelihood method. Acta Crystallogr D Biol. Crystallogr 1997, 53, 240-255. [CrossRef] [PubMed]

53. Nakano, S.; Shinde, A.; Ito, H.; Ito, H.; Kusaka, H. Messenger RNA degradation may be inhibited in sporadic inclusion body myositis. Neurology 2005, 65, 420-425. [CrossRef]

54. Otter, S.; Grimmler, M.; Neuenkirchen, N.; Chari, A.; Sickmann, A.; Fischer, U. A comprehensive interaction map of the human survival of motor neuron (SMN) complex. J. Biol. Chem. 2007, 282, 5825-5833. [CrossRef]

55. Thoma, C.; Ostareck-Lederer, A.; Hentze, M.W. A poly(A) tail-responsive in vitro system for cap- or IRES-driven translation from HeLa cells. Methods Mol. Biol. 2004, 257, 171-180. [CrossRef] [PubMed]

56. Linder, B.; Plottner, O.; Kroiss, M.; Hartmann, E.; Laggerbauer, B.; Meister, G.; Keidel, E.; Fischer, U. Tdrd3 is a novel stress granule-associated protein interacting with the Fragile-X syndrome protein FMRP. Hum. Mol. Genet. 2008, 17, 3236-3246. [CrossRef] [PubMed]

57. Anderson, P.; Kedersha, N. Stress granules: The Tao of RNA triage. Trends Biochem. Sci 2008, 33, 141-150. [CrossRef] [PubMed]

58. Arimoto, K.; Fukuda, H.; Imajoh-Ohmi, S.; Saito, H.; Takekawa, M. Formation of stress granules inhibits apoptosis by suppressing stress-responsive MAPK pathways. Nat. Cell Biol. 2008, 10, 1324-1332. [CrossRef] [PubMed]

59. Deragon, J.M. Distribution, organization an evolutionary history of La and LARPs in eukaryotes. RNA Biol. 2020, 1-9. [CrossRef]

(C) 2020 by the authors. Licensee MDPI, Basel, Switzerland. This article is an open access article distributed under the terms and conditions of the Creative Commons Attribution (CC BY) license (http://creativecommons.org/licenses/by/4.0/). 